\title{
Decoding the Apparent Horizon: Coarse-Grained Holographic Entropy
}

\author{
Netta Engelhardt ${ }^{*}$ \\ Department of Physics, Princeton University, Princeton, New Jersey 08544, USA \\ Aron C. Wall ${ }^{\dagger}$ \\ Institute for Advanced Study, Einstein Drive, Princeton, New Jersey 08540, USA
}

(Received 24 July 2017; published 19 November 2018)

\begin{abstract}
When a black hole forms from collapse in a holographic theory, the information in the black hole interior remains encoded in the boundary. We prove that the area of the black hole's apparent horizon is precisely the entropy associated with coarse graining over the information in its interior, subject to knowing the exterior geometry. This is the maximum holographic entanglement entropy that is compatible with all classical measurements conducted outside of the apparent horizon. We identify the boundary dual to this entropy and explain why it obeys the second law of thermodynamics.
\end{abstract}

DOI: 10.1103/PhysRevLett.121.211301

Introduction.-The second law of thermodynamics states that entropy increases with time. One natural notion of entropy is the von Neumann entropy:

$$
S[\rho]=-\operatorname{tr}(\rho \ln \rho),
$$

where $\rho$ is the density matrix of a quantum system. However, this quantity is conserved under unitary time evolution, in apparent tension with the second law. To obtain an increasing entropy, it is necessary to coarse grain $S$ by "forgetting" certain information, since the vast majority of microscopic data in a thermal system is inaccessible to macroscopic observations. One common coarse-graining method is the maximization of the system's entropy subject to fixed the values of a set of feasible macroscopic measurements $\mathcal{M}(t)$ at a moment in time [1-3]:

$$
S^{\text {coarse }}(t)=\max _{\rho^{\prime}}\left[S\left[\rho^{\prime}\right]: \mathcal{M}(t)\right] .
$$

Assuming that any ordered information inaccessible at early times remains so at later times, $S^{\text {coarse }}$ should increase with time, defining a nontrivial second law.

The most mysterious application of the second law is to black holes. Stationary black holes (e.g., Kerr) have entropy, which is proportional to the area of their horizon $H$ $[4,5]$ :

$$
S_{\mathrm{BH}}=\frac{\operatorname{Area}[H]}{4 G \hbar},
$$

Published by the American Physical Society under the terms of the Creative Commons Attribution 4.0 International license. Further distribution of this work must maintain attribution to the author(s) and the published article's title, journal citation, and DOI. Funded by SCOAP ${ }^{3}$. as suggested by the laws of black hole mechanics [4-7]. However, despite some clues from string theory and other approaches (reviewed in Ref. [8]), it is still unclear in general what microscopic quantum-gravitational degrees of freedom are counted by this entropy. Dynamically evolving black holes such as those formed from stellar collapse are even more controversial, since there are multiple possible definitions of a horizon, e.g., the event horizon and the apparent horizon [9] — and correspondingly, multiple area increase theorems [6,10-14].

In holographic models of quantum gravity, a black hole is dual to some boundary state $\rho$ whose von Neumann entropy $S[\rho]$ can be computed from a compact extremal (HRT) surface in the bulk, as conjectured in Refs. [15,16] and essentially proven in Refs. $[17,18]$ :

$$
S[\rho]=\frac{\operatorname{Area}\left[X_{\mathrm{HRT}}\right]}{4 G \hbar} .
$$

A surface is extremal if its area is unchanged by any first order perturbation to the surface's location; if there is more than one, $X_{\mathrm{HRT}}$ is the one with the minimal area extremal surface (and homologous to the boundary [16,19]). (Because we restrict attention to the entropy of the whole CFT, in this case the HRT surfaces are compact and do not reach the boundary.) This quantity is independent of time, so it is not suitable for describing the entropy increase of a growing black hole. Unitarity of the boundary theory implies that no information is lost, but this is not enough: to account for the increase of black hole entropy, a coarse graining scheme must be specified.

Even though black hole thermodynamics was the original motivation for the holographic principle [20,21], no one has yet given a clear explanation of the role of the black hole horizon as a repository of information about the 
interior. Indeed, it was recently shown [22] that if we know the outcome of all classical measurements $\mathcal{M}(t)$ outside of the event horizon $H$, then $S^{\text {coarse }}<\operatorname{Area}[H] / 4 G \hbar$ : we have access to too much information for our remaining ignorance to be given by the event horizon's area (thus refuting a broad class of proposals relating entropy to area, including [23-26].)

We therefore look for alternatives to the event horizon. An appealing option is the apparent horizon $\mu$, the outermost compact surface (at a moment of time) which is marginally outer trapped [9], i.e., the expansion $\theta_{k} \equiv$ $\nabla_{k} \ln (\operatorname{Area}[n])=0$, where $k$ is a future-outwards null vector, and $n$ is a small pencil of light rays shot out in the $k$ direction from a small neighborhood of a point on $\mu$. In the case of a black hole that forms from collapse, such marginally trapped surfaces form behind the event horizon, even though the HRT surface is the empty set (so that the boundary state is pure).

In this Letter, we give a geometric proof (using classical GR methods in the bulk) that the area of the apparent horizon $\mu$ does play the role of a coarse-grained entropy:

$$
S^{\text {coarse }}=\frac{\operatorname{Area}[\mu]}{4 G \hbar},
$$

where we coarse grain over the region behind the apparent horizon (the "microstates") while holding all classical measurements in the exterior fixed (i.e., we fix all data in the exterior, but working in the classical regime). This makes it plausible that the interior is encoded holographically by a set of independent qubits, one per $4 / \ln 2$ Planck areas, on the apparent horizon (but not the event horizon) [27-30]. Our classical proof explicitly constructs the entropy-maximizing geometry, which would correspond to maximally scrambling all of these qubits. If our result can be extended to the quantum regime (along the lines of Refs. [31-35] it might provide insight into the firewalls paradox [36-39], a puzzle about whether maximally scrambled black holes have an interior. An investigation on areas of noncompact analogues of the apparent horizons will appear in Ref. [40].

Note that although apparent horizons are highly nonunique due to the choice of time slicing, the above construction is valid for each of them.

We also identify the boundary dual to $S^{\text {coarse }}$ of the apparent horizon. This quantity may be computed by maximizing the boundary von Neumann entropy while keeping fixed the outcomes of a set of "simple" experiments performed after a given moment in time. This new entry in the holographic dictionary (which we show is exact to all orders in perturbation theory for near-equilibrium black holes), extends the HRT prescription to a much more general class of bulk surfaces.

Both the bulk and corresponding boundary entropies automatically satisfy the second law. This provides the first valid holographic explanation of the area increase law for black holes.

Outer entropy.-The outer entropy is a coarse-grained entropy that holds fixed the exterior of a codimension-2 surface $\sigma$. We define $O_{W}[\sigma]$, the outer wedge, as the region spacelike outside of $\sigma$ (on the side with the asymptotic boundary). The outer entropy is

$$
S^{(\text {outer })}[\sigma] \equiv \max _{\rho^{\prime}}\left(S\left[\rho^{\prime}\right]: O_{W}[\sigma]\right),
$$

where $\rho^{\prime}$ is any state of the boundary CFT with a classical bulk dual geometry $M^{\prime}$; we choose $\rho^{\prime}$ to maximize the von Neumann entropy $S\left[\rho^{\prime}\right]=X_{\mathrm{HRT}}\left[M^{\prime}\right]$, subject to the constraint that $M^{\prime}$ have the same outer wedge $O_{W}[\sigma]$ as the original classical bulk $M$ dual to $\rho$. Although we have phrased this maximization in terms of the boundary state, note that this can be regarded as a pure bulk construction involving maximizing the area of the HRT surface. The only holographic aspect (in this section) is the identification of an extremal surface lodged inside the black hole with a fine-grained entropy (i.e., the von Neumann entropy). Any theory with such an identification-even one with asymptotically flat boundary conditions (should such a theory exist)-allows the interpretation of $S^{\text {(outer) }}$ as a coarsegrained entropy.

While this coarse-grained entropy can be defined for a general surface $\sigma$, when $\sigma=\mu$, an apparent horizon, we will show that

$$
S^{(\text {outer })}[\mu]=\frac{\operatorname{Area}[\mu]}{4 G \hbar} .
$$

Hence, the area of the apparent horizon has a statistical interpretation as the maximum boundary entropy that is compatible with the geometry of its exterior. This provides a holographic answer to the disputed question: what does the Bekenstein-Hawking entropy of a black hole [41-46] count?

Outline of proof.-Let $k$ (respectively, $\ell$ ) be the orthogonal future-directed null vectors pointing outward (respectively, inward) from a surface. An extremal surface $X$ satisfies $\theta_{k}=\theta_{\ell}=0$. An HRT surface additionally must be the minimal area surface (homologous to the boundary) on some spatial slice $\Sigma$ [47].

An apparent horizon $\mu$ (an outermost marginally trapped surface) satisfies $\theta_{k}=0, \theta_{\ell} \leq 0$, and (generically) $\nabla_{k} \theta_{\ell}<0[48,49]$. We assume that $\mu$ is homologous to the boundary; i.e., there exists a spatial slice $\Sigma$ connecting $\mu$ to the boundary, and moreover that there exists a $\Sigma$ such that the area of any surface circumscribing $\mu$ is larger than the area of $\mu$. These requirements are reasonable for black hole horizons.

In any spacetime, $\operatorname{Area}\left[X_{\mathrm{HRT}}\right] \leq \operatorname{Area}[\mu]$; this can be proven by a simple focusing argument: in a spacetime satisfying the null energy Ccondition $\left(T_{v v} \geq 0\right.$ for any null 
vector $v$ ), a null surface $N_{ \pm k}[\mu]$ shot out along the $\pm k$ direction of $\mu$ has monotonically decreasing area moving away from $\mu$ along $N_{ \pm k}$ in the $+k$ or $-k$ directions, where we truncate the surface when generators intersect [9,50,51]. We extend $N_{ \pm k}$ along its generators to the slice $\Sigma$ on which $X_{\mathrm{HRT}}$ is minimal [47].

$$
\operatorname{Area}[\mu] \geq \operatorname{Area}\left[\Sigma \cap N_{ \pm k}[\mu]\right] \geq \operatorname{Area}\left[X_{\mathrm{HRT}}\right]
$$

Hence, the entropy $S\left[\rho^{\prime}\right]$ cannot exceed $\operatorname{Area}[\mu] / 4 G \hbar$.

To prove that this inequality is saturated, we construct a bulk spacetime $M^{\prime}$ (with the same outer wedge $O_{W}[\mu]$ ) satisfying $\operatorname{Area}\left[X_{\mathrm{HRT}}\right]=\operatorname{Area}[\mu]$. To specify the interior data in $M^{\prime}$, we impose initial data on $N_{-k}$, the null surface fired from $\mu$ in the $-k^{a}$ direction. We choose our initial data so that the surface $N_{-k}$ is stationary; every cross section has the same geometry. (The Appendix shows this construction satisfies the constraint equations, so that a spacetime solution $M^{\prime}$ exists, due to $\nabla_{\ell} \theta_{k}<0$.)

By following $N_{-k}$ far enough, we eventually come to an extremal surface $X$ (see Appendix for details). Since $N_{-k}$ is stationary, Area $[X]=\operatorname{Area}[\mu]$. We can complete the spacetime by requiring it to be invariant under a $C P T$ reflection about $X$ (i.e., we reflect space and time about $X$ while exchanging matter with antimatter). See Fig. 1. The resulting bulk $M^{\prime}$ has two asymptotic boundaries, and therefore represents a pure state (analogous to the thermofield double wormhole construction [52]). When the state $\rho^{\prime}$ is restricted to a single boundary, the entropy $S\left[\rho^{\prime}\right]=$ $X_{\mathrm{HRT}}\left[M^{\prime}\right]$. (Note that the region $O_{W}\left[X_{\mathrm{HRT}}\right]$ agrees with the original bulk geometry dual to $\rho[47,53-57]$.)

Because $N_{-k}$ is stationary and by assumption $\mu$ is minimal on a slice of $O_{W}[\mu]$, we now have an initial data slice $\Sigma$ on which $X$ is the minimal cross section. Any other extremal surface $X^{\prime}$ has greater area than $X$ :

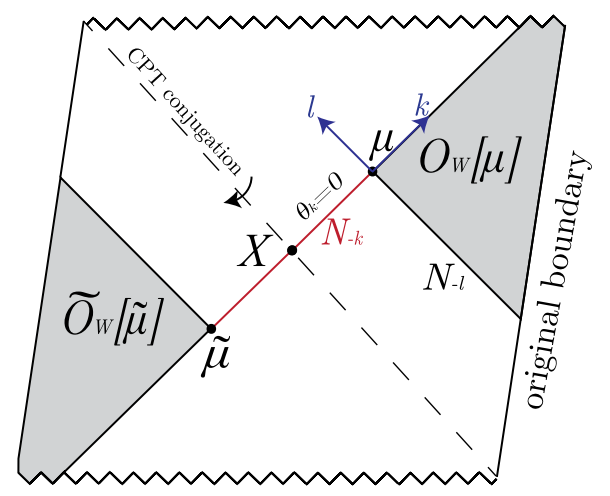

FIG. 1. The coarse-grained spacetime dual to the state $\rho^{\prime}$ with maximal $S\left[\rho^{\prime}\right]$ and fixed $O_{W}[\mu]$ (shaded gray). The null congruence $N_{-k}$ (red) is fired from $\mu$ towards the $-k$ direction and is stationary. The congruence $N_{-l}$, the past boundary of $O_{W}[\mu]$, is fired in the $-\ell$ direction from $\mu$. $X$ is the HRT surface of the coarse-grained spacetime. Tilded quantities represent the $C P T$ mirror reverse.

$$
\operatorname{Area}\left[X^{\prime}\right] \geq \operatorname{Area}\left[\Sigma \cap N_{ \pm k}\left[X^{\prime}\right]\right] \geq \operatorname{Area}[X],
$$

where the first inequality comes from the focusing of a null surface $N_{ \pm k}\left[X^{\prime}\right]$ shot out from $X^{\prime}$. Hence $X=X_{\mathrm{HRT}}$, proving Eq. (7).

Simple entropy.-Thus far, our coarse-grained entropy has been defined from the bulk point of view. We now identify the boundary dual to the outer entropy, which we call the simple entropy, as it relies on "simple operators."

In AdS/CFT, single trace operators on the boundary correspond to locally propagating fields in the bulk. More generally, we expect that the product of a small number of single trace operators also propagates locally in the bulk. However, it is known that sufficiently complicated operators (known as precursors [58,59]) can change the deep bulk region acausally; hence to define a coarse graining that is dual to $O_{W}[\mu]$ we must avoid such complicated operations. We therefore define a simple experiment as a procedure performed after a moment of time $t_{i}$, in which we measure a local operator $\mathcal{O}\left(t>t_{i}\right)$ after having turned on a set of local sources $J\left(t>t_{i}\right)$; we require that these sources propagate causally into the bulk. For classical solutions, we can restrict attention to one-point operators and sources, since the higher-point functions are determined from them. (The "one-point entropy" [26], proposed as a holographic dual to the area of the event horizon, did not allow sources.) To prevent recurrences, we implicitly include a late time cutoff $t_{f}$ prior to exponentially large values of $t$.

The simple entropy is now defined as the maximum entropy of a state $\rho^{\prime}$ compatible with the outcomes of all such simple experiments (i.e., the maximization is done over a subspace of $\rho$ 's that all yield the same outcomes):

$$
S^{(\text {simple })}\left(t_{i}\right)=\max _{\rho^{\prime}}\left(S\left[\rho^{\prime}\right]:\left\langle E^{\dagger} \mathcal{O}(t) E\right\rangle \text { fixed }\right),
$$

where $\rho^{\prime}$ is defined at $t_{i}$, and

$$
E=\mathcal{T} \exp \left(-i \int_{t_{i}}^{t} J\left(t^{\prime}\right) \mathcal{O}_{J}\left(t^{\prime}\right) d t^{\prime}\right)
$$

is the time-ordered insertion of sources $J(t)$ used to prepare the simple experiment by which $\mathcal{O}(t)$ is measured.

A simple experiment, by definition, can only access the subset of the bulk $F\left(t_{i}\right)$ that is to the future of the boundary time $t_{i}$. When the spacetime has a black hole, turning on simple sources can shift the location of any event horizon $H$ in the spacetime [60]. However, the event horizon must always remain outside of any marginally trapped surface (assuming the null energy condition) [9,51]. Therefore, if $\mu$ is a marginally trapped surface on $N\left(t_{i}\right)$, the boundary of $F\left(t_{i}\right)$, a simple experiment can access at most the outer wedge $O_{W}[\mu]$. Note that by causality, turning on simple sources cannot modify the fact that $\mu$ is marginally trapped (a similar argument was given for extremal surfaces in [33]). See Fig. 2(a). It immediately follows that 


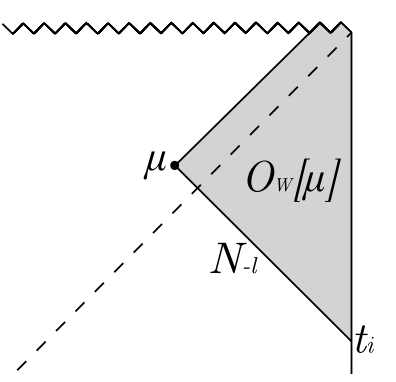

(a)

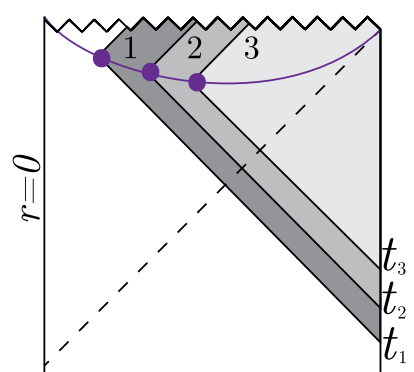

(b)
FIG. 2. (a) We fire a null congruence $N_{-\ell}$ into the bulk from time $t=t_{i}$. The surface $\mu$ is the first cross section of $N_{-\ell}$ with vanishing $k$ expansion. We can recover all the data in $O_{W}[\mu]$, at least when the black hole is near equilibrium, by means of a "simple experiment" performed after time $t_{i}$. (b) A spacelike holographic screen (purple) has increasing area in a spacelike direction, going from 1 to 3 . The corresponding outer wedges are nested, implying that the outer entropy must increase outwards. Similarly, the simple entropy must increase with $t$ from $t_{1}$ to $t_{3}$.

$$
S^{(\text {simple })}\left(t_{i}\right) \geq S^{(\text {outer })}[\mu] \text {. }
$$

If $N\left(t_{i}\right)$ contains more than one marginally trapped surface, we restrict attention the earliest (i.e., outermost) one. This guarantees that $\mu$ is in fact an apparent horizon. We propose that in this case, the inequality (13) is saturated. In other words the simple entropy is the holographic dual of the area of the apparent horizon.

We now show that this is true for a black hole that is approaching thermal equilibrium after time $t_{i}$. We may use the "HKLL" procedure [61-67] to reconstruct the "causal wedge" $C_{W}\left[t_{i}\right]$ of $t_{i}$, i.e., the subset of $F\left(t_{i}\right)$ outside of the event horizon $[68,69]$. If no matter or gravitational radiation were falling across the event horizon $H$, it would be stationary; there would be no separation between $H$ and $\mu$, and we would be done. In order to reconstruct the data in $O_{W}[\mu]$, we must ensure that no matter falls across $H$ after $\mu$.

Since $\mu$ is perturbatively close to the event horizon, Refs. [63,70] allow us to map the matter fields falling across the event horizon to data on the boundary. We can therefore turn these fields "off" by adding suitable sources to the boundary after $t_{i}$. This has the effect of shifting the event horizon to the location of $\mu$, so that $C_{W}\left[t_{i}\right]=O_{W}[\mu]$. (When light rays in $N\left(t_{i}\right)$ intersect before reaching $\mu$, $O_{W}[\mu] \supset C_{W}\left[t_{i}\right]$ since the past boundaries do not coincide. However, $O_{W}[\mu]$ still lies in the domain of dependence of $C_{W}\left[t_{i}\right]$ allowing reconstruction of the full data [71].) This shows that we can use HKLL to reconstruct the spacetime data arbitrarily close to $\mu$. (Although to reconstruct points a distance $\epsilon$ from $\mu$, we need to wait a time of order $\ln \left(\epsilon^{-1}\right)$ for the signal to reach the boundary.) This shows that, order by order in small perturbation to a stationary black hole,

$$
S^{(\text {simple })}\left(t_{i}\right)=\frac{\operatorname{Area}[\mu]}{4 G \hbar}
$$

This is a new entry in the holographic dictionary, which we conjecture also holds for finite deviations from thermality.

An explanation for the second law.-A surface $\mathcal{H}$ foliated by marginally trapped surfaces and satisfying certain regularity conditions obeys an area law: the area of the marginally trapped surfaces increases with evolution along $\mathcal{H}$ [10-14]. In the case where the marginally trapped surfaces foliating $\mathcal{H}$ are apparent horizons, $\mathcal{H}$ must be spacelike [10], and are called trapping horizons [10], dynamical horizons [11,72], or spacelike future holographic screens [13]. The area law for these surfaces says that the area of slices of $\mathcal{H}$ increase going in an outward direction.

The spacelike holographic screen $\mathcal{H}$ is illustrated in Fig. 2(b) in a collapsing black hole, where such objects are ubiquitous. The area increases in outwards evolution along apparent horizon slices of $\mathcal{H}$. The corresponding outer wedges are nested: evolving in the direction of increasing area corresponds to computing the outer entropy of progressively smaller outer wedges. This provides an immediate explanation for why the outer entropy increases along $\mathcal{H}$ : evolution along $\mathcal{H}$ is the equivalent of maximizing the von Neumann entropy with progressively fewer constraints.

From a boundary perspective, the simple entropy increases for much the same reason, since as $t_{i}$ is increased, there are fewer simple experiments available. It may seem odd that the simple entropy also allows measurements to be made at times after $t_{i}$, but this is equivalent to saying that, for a coarsegraining scheme to have a second law, information cannot be discarded if it is going to become available later. (Our very late time cutoff $t_{f}$, which is held constant as $t_{i}$ is increased, prevents us from having to worry about recurrences.)

It is a pleasure to thank $\mathrm{R}$. Bousso, $\mathrm{X}$. Dong, $\mathrm{G}$. Horowitz, J. Maldacena, D. Marolf, F. Pretorius, J. Santos, D. Stanford, H. Verlinde, S. Weinberg, B. White, and E. Witten for helpful discussions. The work of N. E. is supported in part by NSF Grant No. PHY-1620059, while A. W. was supported by the Institute for Advanced Study, the Raymond and Beverly Sackler Foundation Fund, and NSF Grant No. PHY-1314311.

Appendix: Constraint equations.-Since we are imposing data on $N_{-k}$, we need to use the "characteristic initial data formalism" [73-79], which guarantees the existence of a solution (Luk [76] only guarantees a local solution, but then presumably it is possible to deform the characteristic Cauchy slice into a nearby spacelike slice, guaranteeing the existence and uniqueness of $M^{\prime}$ [51].) if we satisfy the following constraint equations on $N_{-k}$ (one for each spacetime dimension $D$ ):

$$
\begin{aligned}
& \nabla_{k} \theta_{k}=-\frac{1}{D-2} \theta_{k}^{2}-\varsigma_{k}^{2}-8 \pi G T_{k k} \\
& \nabla_{k} \chi_{i}=-\theta_{k} \chi_{i}+\left(\frac{D-3}{D-2}\right) \nabla_{i} \theta_{k}-\left(\nabla \cdot \varsigma_{k}\right)_{i}+8 \pi T_{i k}
\end{aligned}
$$


$\nabla_{k} \theta_{\ell}=-\frac{1}{2} \mathcal{R}-2 \nabla \cdot \chi-\theta_{\ell} \theta_{k}+2 \chi^{2}+8 \pi G T_{\ell k}$,

as well as the corresponding junction conditions which require $\theta_{k}, \chi_{i}$, and $\theta_{\ell}$ to be continuous. Here $\varsigma_{k}$ is the shear tensor, which is free data on $N_{-k} ; \mathcal{R}$ is the intrinsic Ricci curvature of cross sections of $N_{-k} ; \chi_{i}$ is a $D-2$ component twist 1 -form gauge field that tells you how much a normal vector gets boosted when transported in the transverse $i$ direction; $T_{a b}$ is the stress tensor. All quantities are defined on constant $v$ slices, where $v$ is an affine parameter defined on each null geodesic of $N_{-k}$, normalized so that $\nabla_{k}=\nabla_{v}$, and $k \cdot \ell=-1$.

We can solve these constraint equations for stationary $N_{-k}$ by stipulating that $\varsigma=\theta_{k}=T_{k k}=T_{k i}=0$, while $\mathcal{R}$, $\chi_{i}, T_{\ell k}$ are constant along $v$. The marginality condition $\theta_{k}[\mu]=0$ ensures continuity of $\theta_{\ell}$ and $\theta_{k}$ on the junction between $N_{-k}$ and $O_{W}[\mu]$. The shear is generically discontinuous across the junction, but that is not a problem for local evolution of the Einstein equation [80,81]. We assume without proof that evolution is possible with AdS boundary conditions.

The above conditions on the stress tensor can be satisfied by reasonable matter fields. For a minimally coupled scalar field $\phi$, take $\phi=$ constant in the $k$ direction; for a Maxwell field $A_{a}$, impose $\nabla_{k} A_{i}=0$ in the gauge $A_{k}=0$. In the Maxwell case there is one additional constraint equation for $\nabla_{k} F_{\ell k}$ that is satisfied if the current $j_{k}=0$.

Because $\mu$ is a apparent horizon, generically $\nabla_{k} \theta_{\ell}<0$ on $N_{-k}$ and $\theta_{\ell}[\mu]<0$. It follows that there exists an extremal cross section $X$ of $N_{-k}$ with $\theta_{\ell}=0$ (and $\left.\theta_{k}=0\right)$. We can solve for the location of $X$ :

$$
0=\theta_{\ell}[\mu]+\theta_{\ell, k} v+\square v+2 \chi \cdot \nabla v,
$$

where $v$ is a function of the transverse directions. There is a unique solution to this equation, with $v<0$ (see Ref. [82]).

To complete our spacetime $M^{\prime}$, we invoked $C P T$ conjugation across the extremal surface $X$. The junction conditions are satisfied at $X$ because $\theta_{\ell}=\theta_{k}=0$ while $\chi_{i}$, $F_{\ell k}, A_{i}$ and $\phi$ are even under $C P T$; for more general matter fields, we expect that $C P T$ invariance ensures that this gluing is always possible.

*nengelhardt@princeton.edu †aroncwall@gmail.com

[1] E. T. Jaynes, Information theory and statistical mechanics, Phys. Rev. 106, 620 (1957).

[2] E. T. Jaynes, Information theory and statistical mechanics. II, Phys. Rev. 108, 171 (1957).

[3] M. Gell-Mann and J. B. Hartle, Quasiclassical coarse graining and thermodynamic entropy, Phys. Rev. A 76, 022104 (2007).

[4] J. D. Bekenstein, Black holes and the second law, Nuovo Cimento Lett. 4, 737 (1972).
[5] S. W. Hawking, Particle creation by black holes, Commun. Math. Phys. 43, 199 (1975).

[6] S. W. Hawking, Gravitational Radiation from Colliding Black Holes, Phys. Rev. Lett. 26, 1344 (1971).

[7] J. M. Bardeen, B. Carter, and S. W. Hawking, The four laws of black hole mechanics, Commun. Math. Phys. 31, 161 (1973).

[8] S. Carlip, Black hole thermodynamics and statistical mechanics, Lect. Notes Phys. 769, 89 (2009).

[9] S. W. Hawking and G. F. R. Ellis, The Large Scale Stucture of Space-Time (Cambridge University Press, Cambridge, England, 1973).

[10] S. A. Hayward, General laws of black hole dynamics, Phys. Rev. 49, 6467 (1994).

[11] A. Ashtekar and B. Krishnan, Dynamical Horizons: Energy, Angular Momentum, Fluxes and Balance Laws, Phys. Rev. Lett. 89, 261101 (2002).

[12] E. Gourgoulhon and J. L. Jaramillo, A $3+1$ perspective on null hypersurfaces and isolated horizons, Phys. Rep. 423, 159 (2006).

[13] R. Bousso and N. Engelhardt, New Area Law in General Relativity, Phys. Rev. Lett. 115, 081301 (2015).

[14] F. Sanches and S. J. Weinberg, Refinement of the Bousso-Engelhardt area law, Phys. Rev. D 94, 021502 (2016).

[15] S. Ryu and T. Takayanagi, Holographic Derivation of Entanglement Entropy from AdS/CFT, Phys. Rev. Lett. 96, 181602 (2006).

[16] V.E. Hubeny, M. Rangamani, and T. Takayanagi, A Covariant holographic entanglement entropy proposal, J. High Energy Phys. 07 (2007) 062.

[17] A. Lewkowycz and J. Maldacena, Generalized gravitational entropy, J. High Energy Phys. 08 (2013) 090.

[18] X. Dong, A. Lewkowycz, and M. Rangamani, Deriving covariant holographic entanglement, J. High Energy Phys. 11 (2016) 028.

[19] M. Headrick and T. Takayanagi, A Holographic proof of the strong subadditivity of entanglement entropy, Phys. Rev. D 76, 106013 (2007).

[20] G. 't Hooft, Dimensional reduction in quantum gravity, Conf. Proc. C930308, 284 (1993).

[21] L. Susskind, The World as a hologram, J. Math. Phys. (N.Y.) 36, 6377 (1995).

[22] N. Engelhardt and A. C. Wall, No simple dual to the causal holographic information?, J. High Energy Phys. 04 (2017) 134.

[23] R. D. Sorkin, The statistical mechanics of black hole thermodynamics, arXiv:gr-qc/9705006.

[24] E. Bianchi and R. C. Myers, On the architecture of spacetime geometry, Classical Quantum Gravity 31, 214002 (2014).

[25] B. Freivogel and B. Mosk, Properties of causal holographic information, J. High Energy Phys. 09 (2013) 100.

[26] W. R. Kelly and A. C. Wall, Coarse-grained entropy and causal holographic information in AdS/CFT, J. High Energy Phys. 03 (2014) 118.

[27] R. Bousso, The holographic principle for general backgrounds, Classical Quantum Gravity 17, 997 (2000).

[28] R. Bousso, The holographic principle, Rev. Mod. Phys. 74, 825 (2002). 
[29] F. Sanches and S. J. Weinberg, Holographic entanglement entropy conjecture for general spacetimes, Phys. Rev. D 94, 084034 (2016).

[30] Y. Nomura, N. Salzetta, F. Sanches, and S. J. Weinberg, Toward a holographic theory for general spacetimes, Phys. Rev. D 95, 086002 (2017).

[31] A. C. Wall, The generalized second law implies a quantum singularity theorem, Classical Quantum Gravity 30, 165003 (2013).

[32] R. Bousso and N. Engelhardt, Generalized second law for cosmology, Phys. Rev. D 93, 024025 (2016).

[33] N. Engelhardt and A. C. Wall, Quantum extremal surfaces: Holographic entanglement entropy beyond the classical regime, J. High Energy Phys. 01 (2015) 073.

[34] T. Faulkner, A. Lewkowycz, and J. Maldacena, Quantum corrections to holographic entanglement entropy, J. High Energy Phys. 11 (2013) 074.

[35] X. Dong and A. Lewkowycz, Entropy, extremality, euclidean variations, and the equations of motion, J. High Energy Phys. 01 (2018) 081.

[36] A. Almheiri, D. Marolf, J. Polchinski, and J. Sully, Black holes: Complementarity or firewalls?, J. High Energy Phys. 02 (2013) 062.

[37] A. Almheiri, D. Marolf, J. Polchinski, D. Stanford, and J. Sully, An apologia for firewalls, J. High Energy Phys. 09 (2013) 018.

[38] S. D. Mathur and C. J. Plumberg, Correlations in Hawking radiation and the infall problem, J. High Energy Phys. 09 (2011) 093,

[39] S. L. Braunstein, S. Pirandola, and K. Życzkowski, Better Late than Never: Information Retrieval from Black Holes, Phys. Rev. Lett. 110, 101301 (2013).

[40] D. Marolf and B. White, Marginally trapped surfaces and AdS/CFT, J. High Energy Phys. 02 (2018) 049.

[41] T. Jacobson, On the nature of black hole entropy, AIP Conf. Proc. 493, 85 (1999).

[42] R. D. Sorkin, Ten theses on black hole entropy, Stud. Hist. Phil. Sci. B 36, 291 (2005).

[43] T. Jacobson, D. Marolf, and C. Rovelli, Black hole entropy: Inside or out?, Int. J. Theor. Phys. 44, 1807 (2005).

[44] B. Freivogel, V. E. Hubeny, A. Maloney, R. C. Myers, M. Rangamani, and S. Shenker, Inflation in AdS/CFT, J. High Energy Phys. 03 (2006) 007.

[45] S. D. H. Hsu and D. Reeb, Unitarity and the Hilbert space of quantum gravity, Classical Quantum Gravity 25, 235007 (2008).

[46] D. Marolf, Black holes, AdS, and CFTs, Gen. Relativ. Gravit. 41, 903 (2009).

[47] A. C. Wall, Maximin surfaces, and the strong subadditivity of the covariant holographic entanglement entropy, Classical Quantum Gravity 31, 225007 (2014).

[48] L. Andersson and J. Metzger, The area of horizons and the trapped region, Commun. Math. Phys. 290, 941 (2009).

[49] M. Mars, Stability of marginally outer trapped surfaces and geometric inequalities, Fundam. Theor. Phys. 177, 191 (2014).

[50] R. Penrose, Gravitational Collapse and Space-Time Singularities, Phys. Rev. Lett. 14, 57 (1965).
[51] R. M. Wald, General Relativity (University of Chicago Press, Chicago, 1984).

[52] J. M. Maldacena, Eternal black holes in anti-de Sitter, J. High Energy Phys. 04 (2003) 021.

[53] B. Czech, J. L. Karczmarek, F. Nogueira, and M. Van Raamsdonk, The gravity dual of a density matrix, Classical Quantum Gravity 29, 155009 (2012).

[54] M. Headrick, V. E. Hubeny, A. Lawrence, and M. Rangamani, Causality \& holographic entanglement entropy, J. High Energy Phys. 12 (2014) 162.

[55] D. L. Jafferis, A. Lewkowycz, J. Maldacena, and S. J. Suh, Relative entropy equals bulk relative entropy, J. High Energy Phys. 06 (2016) 004.

[56] X. Dong, D. Harlow, and A. C. Wall, Bulk Reconstruction in the Entanglement Wedge in AdS/CFT, Phys. Rev. Lett. 117, 021601 (2016).

[57] T. Faulkner and A. Lewkowycz, Bulk locality from modular flow, J. High Energy Phys. 07 (2017) 151.

[58] J. Polchinski, L. Susskind, and N. Toumbas, Negative energy, superluminosity and holography, Phys. Rev. D 60, 084006 (1999), Expanded version replacing earlier hep-th 9902182.

[59] B. Freivogel, S. B. Giddings, and M. Lippert, Toward a theory of precursors, Phys. Rev. D 66, 106002 (2002).

[60] S. H. Shenker and D. Stanford, Black holes and the butterfly effect, J. High Energy Phys. 03 (2014) 067.

[61] A. Hamilton, D. N. Kabat, G. Lifschytz, and D. A. Lowe, Local bulk operators in AdS/CFT: A boundary view of horizons and locality, Phys. Rev. D 73, 086003 (2006).

[62] A. Hamilton, D. N. Kabat, G. Lifschytz, and D. A. Lowe, Holographic representation of local bulk operators, Phys. Rev. D 74, 066009 (2006).

[63] I. Heemskerk, D. Marolf, J. Polchinski, and J. Sully, Bulk and transhorizon measurements in AdS/CFT, J. High Energy Phys. 10 (2012) 165.

[64] V. Balasubramanian, P. Kraus, and A. E. Lawrence, Bulk versus boundary dynamics in anti-de Sitter space-time, Phys. Rev. D 59, 046003 (1999).

[65] V. Balasubramanian, P. Kraus, A. E. Lawrence, and S. P. Trivedi, Holographic probes of anti-de Sitter space-times, Phys. Rev. D 59, 104021 (1999).

[66] T. Banks, M. R. Douglas, G. T. Horowitz, and E. J. Martinec, AdS dynamics from conformal field theory, arXiv:hep-th/9808016.

[67] I. Bena, On the construction of local fields in the bulk of $\operatorname{AdS}(5)$ and other spaces, Phys. Rev. D 62, 066007 (2000).

[68] R. Bousso, S. Leichenauer, and V. Rosenhaus, Light-sheets and AdS/CFT, Phys. Rev. D 86, 046009 (2012).

[69] V.E. Hubeny and M. Rangamani, Causal holographic information, J. High Energy Phys. 06 (2012) 114.

[70] D. Kabat, G. Lifschytz, and D. A. Lowe, Constructing local bulk observables in interacting AdS/CFT, Phys. Rev. D 83, 106009 (2011).

[71] N. Engelhardt and A. Wall (to be published).

[72] A. Ashtekar and G. J. Galloway, Some uniqueness results for dynamical horizons, Adv. Theor. Math. Phys. 9, 1 (2005). 
[73] A. D. Rendall, Reduction of the characteristic initial value problem to the Cauchy problem and its applications to the Einstein equations, Proc. R. Soc. A 427, 221 (1990).

[74] P. R. Brady, S. Droz, W. Israel, and S. M. Morsink, Covariant double null dynamics: $(2+2)$ splitting of the Einstein equations, Classical Quantum Gravity 13, 2211 (1996).

[75] Y. Choquet-Bruhat, P. T. Chrusciel, and J. M. MartinGarcia, The Cauchy problem on a characteristic cone for the Einstein equations in arbitrary dimensions, Ann. Inst. Henri Poincaré 12, 419 (2011).

[76] J. Luk, On the local existence for the characteristic initial value problem in general relativity, arXiv:1107.0898.

[77] P. T. Chrusciel, The existence theorem for the general relativistic Cauchy problem on the light-cone, SIGMA 2, e10 (2014).
[78] P. T. Chrusciel and T.-T. Paetz, The many ways of the characteristic Cauchy problem, Classical Quantum Gravity 29, 145006 (2012).

[79] P. T. Chruściel and T.-T. Paetz, Characteristic initial data and smoothness of Scri. I. Framework and results, Ann. Inst. Henri Poincaré 16, 2131 (2015).

[80] J. Luk and I. Rodnianski, Local propagation of impulsive gravitational waves, Commun. Pure Appl. Math. 68, 511 (2015).

[81] J. Luk and I. Rodnianski, Nonlinear interaction of impulsive gravitational waves for the vacuum Einstein equations, arXiv:1301.1072.

[82] L. Andersson, M. Mars, and W. Simon, Local Existence of Dynamical and Trapping Horizons, Phys. Rev. Lett. 95, 111102 (2005). 